\title{
Separation and weak separation on Riemann surfaces
}

\author{
Junichiro NARITA
}

(Received June 11, 1997)

\begin{abstract}
We show some necessary and sufficient conditions for weak separation by an algebra $A$ of analytic functions on a Riemann surface $R$. One of these equivalent conditions is the following. There exists a sequence of relatively compact open sets $\left\{D_{n}\right\}$ in $R$ such that (i) $\partial D_{n}$ is connected, (ii) $\bar{D}_{1} \subset \bar{D}_{2} \subset \bar{D}_{3} \subset \cdots$, (iii) $R=\cup \bar{D}_{n}$, and (iv) $A$ separates the points of a neighborhood of $\partial D_{n}$.
\end{abstract}

Key words: weak separation, algebra of analytic functions, Riemann surface.

\section{Introduction}

Let $R$ be a Riemann surface, and let $A$ be an algebra of analytic functions on $R$. We always assume that $A$ contains constant functions. We say that points $p$ and $q$ of $R$ are separated by $A$ if there is a function $f$ in $A$ such that $f(p) \neq f(q)$, and when any pair of distinct points are separated by $A$, we say that the algebra $A$ separates the points of $R$. For functions $f$ and $g$ in $A$ such that $g \not \equiv 0,(f / g)$ is a meromorphic function and so we can consider the value $(f / g)(p)$ at any point $p$ of $R$. According to Royden [4] we say that points $p$ and $q$ of $R$ are weakly separated by $A$ if there are functions $f$ and $g$ in $A$ as above such that $(f / g)(p) \neq(f / g)(q)$, and when any pair of distinct points are weakly separated by $A$, we say that the algebra $A$ weakly separates the points of $R$.

On the other hand, in Gamelin-Hayashi [2] it was defined that $A$ weakly separates the points of $R$ if there is a discrete subset $\Lambda$ of $R$ such that $A$ separates the points of $R \backslash \Lambda$ in case $A$ is the algebra of bounded analytic functions $H^{\infty}(R)$. These two definitions for weak separation coincides each other.

In this paper we study some necessary and sufficient conditions for weak separation, and show that separation on a rather narrow set means weak separation on $R$. We also include a proof of equivalence of two definitions for weak separation. It will be convenient since the proof is not given in [2]. For the moment we use the terminology "weak separation" in the sense of 
Royden.

\section{Preparation}

We want to use the Royden's resolution $\tilde{R}$ of $R$ with respect to $A$ and the canonical map $\varphi: R \rightarrow \tilde{R}$, and also some lemmas which are used to construct $\tilde{R}$ in Royden [4]. Following lemmas and proposition are implicitly included in Royden [3]. See also Bishop [1]. We include the proof for the sake of convenience.

Lemma 1 If points $p, q$ of $R$ are weakly separated by $A$, then there are neighborhoods $U$ of $p$ and $V$ of $q$ such that $A$ separates any pair of points $\left(p^{\prime}, q^{\prime}\right)$ in $U \times V$ except $(p, q)$.

Proof. Let $f$ and $g$ be functions in $A$ such that $(f / g)(p) \neq(f / g)(q)$. If $f(p) \neq f(q)$ or $g(p) \neq g(q)$ then the conclusion easily follows, so we can assume that $f(p)=f(q)$ and $g(p)=g(q)$. Then $(f / g)(p) \neq(f / g)(q)$ can occur only when $f(p)=f(q)=g(p)=g(q)=0$. As $g$ is not identically 0 , there exist neighborhoods $U$ of $p$ and $V$ of $q$ such that $g \neq 0$ in $(U \backslash\{p\}) \cup$ $(V \backslash\{q\})$ and $(f / g)(U) \cap(f / g)(V)=\emptyset$. Then any pair of points $\left(p^{\prime}, q^{\prime}\right)$ in $(U \times V) \backslash\{(p, q)\}$ are separated by functions $f$ or $g$ in $A$.

For a point $p$ of $R$, let

$$
M(p)=\{f / g: f, g \in A, g \not \equiv 0,(f / g)(p)=0\}
$$

and let $\nu(p)$ be the minimal order of meromorphic functions in $M(p)$ at $p$.

Lemma 2 For a point $p$ of $R$, let $h$ be a function in $M(p)$ with order $\nu(p)$ at $p$. Then for any function $f$ in $A$, there exists a neighborhood $U$ of $p$ such that $f$ is represented as

$$
f=\sum_{n=0}^{\infty} c_{n} h^{n}
$$

in $U$.

Proof. By some local coordinate $(U, z)$ with $z(p)=0, h$ can be represented as $h=z^{\nu(p)}$ and

$$
f=\sum_{m=0}^{\infty} a_{m} z^{m}
$$


in $U$. We want to show that the set $\left\{m: a_{m} \neq 0, m\right.$ is not a multiple of $\nu(p)\}$ is empty. If not, let $\mathrm{s}$ be the smallest number of this set, and let $t$ be the integer with $t \nu(p)<s<(t+1) \nu(p)$. Then

$$
\frac{f-\sum_{k=0}^{t} a_{k \nu(p)} h^{k}}{h^{t}}=\frac{f-\sum_{k=0}^{t} a_{k \nu(p)} z^{k \nu(p)}}{z^{t \nu(p)}}=a_{s} z^{s-t \nu(p)}+\cdots
$$

is an element of $M(p)$ and the order of this function at $p$ is less than $\nu(p)$. This is a contradiction.

Lemma 3 Suppose that A weakly separates the points of $R$. Then for any point $p$ of $R$, there exists a neighborhood $U$ of $p$ such that $A$ separates the points of $U$.

Proof. Let $U$ and $h$ be as in the proof of Lemma 2. Lemma 2 shows that a pair of points in $U$ are not weakly separated by $A$ if these points are not separated by $h$. Since $h=z^{\nu(p)}$, it must be that $\nu(p)=1$. Then $h$ itself separates the points of $U$.

Let $h=f / g$ with $f, g \in A$. By replacing $U$ with a smaller neighborhood of $p$ if necessary, we can assume that $f \neq 0$ and $g \neq 0$ in $U \backslash\{p\}$. Then any pair of points in $U$ are separated by functions $f$ or $g$ in $A$.

The Royden's resolution $\tilde{R}$ of $R$ with respect to $A$ and the cannonical map $\varphi: R \rightarrow \tilde{R}$ is defined in [4] in terms of the homomorphisms of algebras. Here $\tilde{R}$ is a Riemann surface and $\varphi$ is an analytic map satisfying that $\varphi(p)=\varphi(q)$ for $p, q \in R$ if and only if there exist non-constant analytic maps $\rho$ and $\sigma$ from a neighborhood of $p$ and $q$ respectively into the complex plane satisfying that $\rho(p)=0, \sigma(q)=0$, and every $f$ in $A$ takes a same value on $\rho^{-1}(z) \cup \sigma^{-1}(z)$ for any complex number $z$ in the images of $\rho$ or $\sigma$.

We use only this property of $\tilde{R}$ and $\varphi$ in the proof of following proposition, and so we may use this equivalence relation $p \sim q$ to define a Riemann surface $R / \sim$, which is enough for the purpose of this paper, although $\varphi(R)=R / \sim$ is a subsurface of the Royden's resolution $\tilde{R}$ in general.

Proposition 1 For $p$ and $q$ of $R, \varphi(p)=\varphi(q)$ if and only if $p$ and $q$ are not weakly separated by $A$. Especially, the map $\varphi$ is injective on $R$ if and only if $A$ weakly separates the points of $R$.

Proof. If $\varphi(p)=\varphi(q)$, then $A$ does not separate $\rho^{-1}(z)$ and $\sigma^{-1}(z)$ for any $z$ and so by Lemma 1, $A$ does not weakly separate $p$ and $q$.

For the reverse implication, we assume $p$ and $q$ are not weakly separated 
by $A$. Let $h_{p}, h_{q}$ be functions in $M(p), M(q)$ respectively as in the statement of Lemma 2. Since $p$ and $q$ are not weakly separated, $h_{q}(p)=h_{p}(p)=0$, $h_{p}(q)=h_{q}(q)=0$, and $\left(h_{p} / h_{q}\right)(p)=\left(h_{p} / h_{q}\right)(q)$. First two equations imply $h_{q} \in M(p), h_{p} \in M(q)$ and so $\left(h_{p} / h_{q}\right)(p) \neq 0,\left(h_{p} / h_{q}\right)(q) \neq \infty$. Hence $h_{p}$ and $h_{q}$ have the same order at $q$, and so we can take the same function $h=h_{p}$ in Lemma 2 for both $p$ and $q$.

For any $f \in A$, there are neighborhoods $U$ of $p$ and $V$ of $q$ such that $f=$ $\sum_{n=0}^{\infty} a_{n} h^{n}$ in $U$ and $f=\sum_{n=0}^{\infty} b_{n} h^{n}$ in $V$. If $a_{n}=b_{n}$ for $n=0,1, \ldots, k-1$, then the function

$$
\frac{f-\sum_{n=0}^{k-1} a_{n} h^{n}}{h^{k}}
$$

is a member of quotient field of $A$ and takes values $a_{k}$ at $p$ and $b_{k}$ at $q$. So $a_{k}=b_{k}$ and this shows that $a_{n}=b_{n}$ for all $n$. Therefore, if we take $\rho=\sigma=h, f$ takes a same value on $\rho^{-1}(z) \cup \sigma^{-1}(z)=h^{-1}(z)$ for any complex number $z$ in $h(U \cup V)$.

\section{Main Theorem}

For two sets $U$ and $E$ in $R$, We say that $A$ is separating on $U$ with respect to $E$ if every point in $U$ is separated by $A$ from any other point in $U \cup E$.

Theorem 1 Let $A$ be an algebra of analytic functions on a Riemann surface $R$. Then the following four conditions are equivalent.

(a) A weakly separates the points of $R$.

(b) There exists a discrete subset $\Lambda$ of $R$ such that $A$ separates the points of $R \backslash \Lambda$.

(c) There exists a sequence of compact sets $\left\{K_{n}\right\}$ in $R$ such that (i) $K_{1} \subset K_{2} \subset K_{3} \subset \cdots$, (ii) $R=\cup K_{n}$, and (iii) $A$ is separating on a neighborhood of $\partial K_{n}$ with respect to $K_{n}$.

(d) There exists a sequence of relatively compact open sets $\left\{D_{n}\right\}$ in $R$ such that (i) $\partial D_{n}$ is connected, (ii) $\bar{D}_{1} \subset \bar{D}_{2} \subset \bar{D}_{3} \subset \cdots$, (iii) $R=\cup \bar{D}_{n}$, and (iv) A separates the points of a neighborhood of $\partial D_{n}$.

Proof. $\quad(a) \Rightarrow(b)$ : Let

$$
\Gamma=\{(p, q) \in R \times R: p \neq q, p \text { and } q \text { are not separated by } A\} .
$$

By Lemma 1 and Lemma 3, $\Gamma$ is a discrete subset of $R \times R$. Let $\left\{R_{n}\right\}$ be 
an exhaustion of $R$ by relatively compact subregions $R_{n}$ of $R$. For $p \in R$, let $\chi(p)=\min \left\{n: p \in R_{n}\right\}$ and we set

$$
\begin{aligned}
\Lambda=\{p \in R: \text { there exists a } q \in R \\
\text { such that }(p, q) \in \Gamma \text { and } \chi(q) \leq \chi(p)\} .
\end{aligned}
$$

First, we show that $A$ separates the points of $R \backslash \Lambda$. If not, there exists a pair of points $p, q \in R \backslash \Lambda$ which are not separated by $A$, so $(p, q) \in \Gamma$. Then either $p$ or $q$ is a member of $\Lambda$ according to $\chi(q) \leq \chi(p)$ or $\chi(p) \leq \chi(q)$. This is a contradiction.

Next, we show that $\Lambda$ is a discrete subset of $R$. If not, there exists a sequence $\left\{p_{m}\right\}$ of points in $\Lambda$ such that $\left\{p_{m}\right\}$ converges to a point $p$ in $R$. Then all points of $\left\{p_{m}\right\}$ are contained in an $R_{n}$. By the definition of $\Lambda$, for each $p_{m}$ there exists a point $q_{m} \in R$ such that $\left(p_{m}, q_{m}\right) \in \Gamma$ and $q_{m}$ is also contained in $R_{n}$. Since $R_{n}$ is relatively compact, there is a subsequence of $\left\{\left(p_{m}, q_{m}\right)\right\}$ which converges to a point in $R \times R$. This contradicts the fact that $\Gamma$ is a discrete subset of $R \times R$.

(b) $\Rightarrow(\mathrm{d})$ : We can take an exhaustion $\left\{R_{n}\right\}$ of $R$ such that $\partial R_{n}$ consists of finite number of smooth Jordan closed curves and $\partial R_{n} \cap \Lambda=\emptyset$ for all $n$. We can also join every component of $\partial R_{n}$ by finite number of disjoint smooth Jordan arcs in $R_{n}$ without passing $\Lambda$. Let $L_{n}$ be the union of these Jordan arcs. Then $D_{n}=R_{n} \backslash L_{n}$ satisfies the conditions of (d).

(c) $\Rightarrow$ (a): We use the Royden's resolution $\tilde{R}$ of $R$ with respect to $A$ and the canonical map $\varphi: R \rightarrow \tilde{R}$. It suffices to show that $\varphi$ is injective on each $K_{n}$.

First we show that there exists a neighborhood $V$ of $\varphi\left(\partial K_{n}\right)$ such that for $w \in V$, the number of points in $\varphi^{-1}(w) \cap K_{n}$ is 1 or 0 . Let $U$ be a neighborhood of $\partial K_{n}$ such that $A$ is separating on $U$ with respect to $K_{n}$. Since $\varphi$ is an open mapping, $\varphi(U)$ is a neighborhood of $\varphi\left(\partial K_{n}\right)$. Let $w \in \varphi(U)$ and $p \in U$ be such as $\varphi(p)=w$. If there exists another point $q \in K_{n}, \varphi(q)=w$, then $p$ and $q$ are not separated by $A$ which contradicts the assumption. Hence $V=\varphi(U)$ suffices our request.

Now we show that $\varphi$ is injective on int $K_{n}$ by reduction to absurdity. So we assume that there exist points $a, b \in \operatorname{int} K_{n}$ such that $a \neq b$ and $\varphi(a)=\varphi(b)$. If $\varphi(a) \in \varphi(S)$ where $S=\left\{p \in K_{n}: \frac{d \varphi}{d \zeta}(p)=0\right\}$ (the set of singular points of the map $\varphi)$, we can take $c \notin \varphi(S)$ near $\varphi(a)$ and $\tilde{a} \in \varphi^{-1}(c) \cap \operatorname{int} K_{n}$ near $a$ and $\tilde{b} \in \varphi^{-1}(c) \cap \operatorname{int} K_{n}$ near $b$ so that $\tilde{a} \neq \tilde{b}$. Hence we can assume that $\varphi(a) \notin \varphi(S)$. 
We can join $\varphi(a)$ with a point $x \in V$ by a Jordan $\operatorname{arc} \gamma$ in $\tilde{R} \backslash(\varphi(S) \cup$ $\left.\varphi\left(\partial K_{n}\right)\right)$. In fact we can join $\varphi(a)$ with any point $y \in V \backslash \varphi(S)$ by an Jordan arc $\tilde{\gamma}$ in $\tilde{R} \backslash \varphi(S)$ with the equation $u:[0,1] \rightarrow \tilde{R}, u(0)=\varphi(a), u(1)=y$. If $\tilde{\gamma} \cap \varphi\left(\partial K_{n}\right)=\emptyset$, we can take $x=y$ and $\gamma=\tilde{\gamma}$. If $\tilde{\gamma} \cap \varphi\left(\partial K_{n}\right) \neq \emptyset$, let $t_{0}=\min \left\{t: u(t) \in \varphi\left(\partial K_{n}\right)\right\}$ and take $t_{1}$ such that $u\left(t_{1}\right) \in V$ and $t_{1}<t_{0}$. Then we can take $x=u\left(t_{1}\right)$ and the subarc $\gamma$ of $\tilde{\gamma}$ for $0 \leq t \leq t_{1}$.

By usual lifting argument, we see that there exist arcs $\gamma_{a}$ and $\gamma_{b}$ in $R$ with initial points $a$ and $b$ respectively, such that $\varphi\left(\gamma_{a}\right)=\varphi\left(\gamma_{b}\right)=\gamma$ and $\gamma_{a} \cap \gamma_{b}=\emptyset$. Since $\gamma_{a}$ and $\gamma_{b}$ do not meet $\partial K_{n}$, these are contained in int $K_{n}$. Accordingly $\varphi^{-1}(x) \cap K_{n}$ contains at least two points $\varphi^{-1}(x) \cap \gamma_{a}$ and $\varphi^{-1}(x) \cap \gamma_{b}$. This contradicts $x \in V$ and so we see that $\varphi$ is injective on int $K_{n}$. This with the assumptin of (c) shows that $\varphi$ is injective on $K_{n}$.

(d) $\Rightarrow$ (c): We again use the Royden's resolution $\tilde{R}$ of $R$ and $\varphi: R \rightarrow$ $\tilde{R}$. Let $U$ be a neighborhood of $\partial D_{n}$ such that $A$ separates the points of $U$. We can take an arcwise connected compact set $B$ with $\partial D_{n} \subset \operatorname{int} B \subset B \subset$ $U$. For example, we can cover $\partial D_{n}$ by finite number of coordinate disks $V_{m}$ with $\bar{V}_{m} \subset U$ and $V_{m} \cap \partial D_{n} \neq \emptyset$. Then $\cup V_{m}$ is an open connected set and we can take $B=\overline{\cup V_{m}}$ as an arcwise connected compact set.

We want to show that $A$ is separating on $B$ with respect to $D_{n}$ by reduction to absurdity. If not, there exists a point $p \in D_{n} \backslash B$ with $\varphi(p) \in$ $\varphi(B)$. Let $E$ be a component of $\varphi^{-1}(\varphi(B)) \cap\left(D_{n} \cup B\right)$ containing $p$. As $\varphi$ is injective on $U, \varphi(U \backslash B)$ does not meet $\varphi(B)$ and so $\varphi^{-1}(\varphi(B)) \cap\left(D_{n} \cup B\right)$ is contained in the union of mutually disjoint compact sets $D_{n} \backslash U$ and $B$. This shows that $E \subset D_{n} \backslash U \subset D_{n} \backslash B$.

Now we can use lifting argument to show that $\varphi(E)=\varphi(B)$. In fact, for any point $w$ in $\varphi(B)$, we can join $\varphi(p)$ and $w$ by an $\operatorname{arc} \gamma$ in $\varphi(B)$, and we can take a maximal arc $\gamma_{p}$ in $R$ with initial point $p$ such that $\varphi\left(\gamma_{p}\right) \subset \gamma$. If $\varphi\left(\gamma_{p}\right)$ is a proper subset of $\gamma$, then the arc $\gamma_{p}$ continues to the outside of the set $D_{n}$, and $\gamma_{p} \cap \partial D_{n} \neq \emptyset$. Hence, the set $E$ intersects with the set $B$, a contradiction. Thus, $\varphi\left(\gamma_{p}\right)=\gamma$. Then $w \in \varphi\left(\gamma_{p}\right) \subset \varphi(E)$ and so $\varphi(E)=\varphi(B)$.

From $E \subset D_{n}$ and $\partial D_{n} \subset B$, it follows that $\varphi\left(\partial D_{n}\right) \subset \varphi(B)=\varphi(E) \subset$ $\varphi\left(D_{n}\right)$. For any function $f$ in $A$, we can take an analytic function $\tilde{f}$ on $\tilde{R}$ such that $f=\tilde{f} \circ \varphi$. Then $f\left(\partial D_{n}\right)=\tilde{f}\left(\varphi\left(\partial D_{n}\right)\right) \subset \tilde{f}\left(\varphi\left(D_{n}\right)\right)=f\left(D_{n}\right)$ and by the maximal modulus principle, $f$ must be a constant function. This contradicts the assumption of (d), and we conclude that $A$ is separating on $B$ with respect to $D_{n}$. 
Let $K_{n}=\bar{D}_{n}$. Since $\partial K_{n} \subset \partial D_{n} \subset$ int $B \subset B$ and $K_{n} \cup \operatorname{int} B \subset D_{n} \cup B$, conditions of (c) are satisfied if we take int $B$ as a neighborhood of $\partial K_{n}$.

The condition (i) " $\partial D_{n}$ is connected" of (d) in Theorem 1 can not be removed, and also we can not remove "a neighborhood of" in the condition (iv) of (d).

To show this we use a Riemann surface $R$ which is known as Myrberg's example $([3])$, and we take $A$ as the algebra of bounded analytic functions $H^{\infty}(R)$. Let $a_{n}, b_{n}$ be two sequences of real numbers such that $0<a_{n+1}<$ $b_{n+1}<a_{n}<b_{n}(n=1,2, \ldots)$ and $\lim _{n \rightarrow \infty} a_{n}=\lim _{n \rightarrow \infty} b_{n}=0$. We define a Riemann surface $R$ as a two sheeted unbounded covering surface of punctured disk $\Delta_{0}=\{0<|z|<1\}$ which has branch points over $\left\{a_{n}\right\}$ and $\left\{b_{n}\right\}$. Let $\pi: R \rightarrow \Delta_{0}$ be a projection, and let $C_{r}=\{|z|=r\}$. We also assume that $\pi^{-1}\left(C_{r}\right)$ is connected for $a_{n} \leq r \leq b_{n}(n=1,2, \ldots)$ and $\pi^{-1}\left(C_{r}\right)$ has two components for $b_{n+1}<r<a_{n}(n=1,2, \ldots)$ and for $b_{1}<r<1$. It is known that every bounded analytic function on $R$ takes a same value on $\pi^{-1}(z)$ for $z \in \Delta_{0}$, and so $H^{\infty}(R)$ can not weakly separates the points of $R$.

We can take connected open sets $\left\{D_{n}\right\}$ in $R$ such that $\partial D_{n}$ has four components and each component is a component of $\pi^{-1}\left(C_{r}\right)$ for $r=c_{n}, d_{n}, s_{n}, t_{n}$ respectively, where $b_{n+1}<c_{n}<d_{n}<a_{n}$ and $b_{1}<s_{n}<t_{n}<s_{n+1}<t_{n+1}<$ $1(n=1,2, \ldots), \lim _{n \rightarrow \infty} s_{n}=\lim _{n \rightarrow \infty} t_{n}=1$. Note that we must take components of $\pi^{-1}\left(C_{r}\right)$ on "different sheets" of $R$ for $r=c_{n}$ and $r=d_{n}$, and also for $r=s_{n}$ and $r=t_{n}$. The conditions (ii) and (iii) of (d) are satisfied by the construction, and (iv) is satisfied since $H^{\infty}(R)$ contains the function $z \circ \pi$ where $z$ is the coordinate function on $\Delta_{0}$. Now all conditions of (d) are satisfied except (i).

For another example which shows necessity of "a neighborhood of" in the condition (iv) of (d), we modify the Riemann surface $R$ such as $R$ has branch points also over $\left\{s_{n}\right\}$ and $\left\{t_{n}\right\}$. Again every bounded analytic function on $R$ takes a same value on $\pi^{-1}(z)$ for $z \in \Delta_{0}$. Let $\Gamma_{n, 1}$ be a subset of $\pi^{-1}\left(C_{a_{n}}\right)$ which form a closed Jordan curve, and let $\Gamma_{n, 2}$ be another closed Jordan curve on $R$ such that $\pi\left(\Gamma_{n, 2}\right)$ is the circle whose diameter is the segment $\left[-b_{n}, a_{n}\right]$ and such that $R \backslash\left(\Gamma_{n, 1} \cup \Gamma_{n, 2}\right)$ has no relatively compact components. We take $\Gamma_{n, 3}$ and $\Gamma_{n, 4}$ in the same manner, such as $\Gamma_{n, 3} \subset \pi^{-1}\left(C_{s_{n}}\right)$ and $\pi\left(\Gamma_{n, 4}\right)$ is the circle whose diameter is the 
segment $\left[-t_{n}, s_{n}\right]$. Now we can take connected open sets $\left\{\tilde{D}_{n}\right\}$ in $R$ such that $\partial \tilde{D}_{n}$ has two components $\Gamma_{n, 1} \cup \Gamma_{n, 2}$ and $\Gamma_{n, 3} \cup \Gamma_{n, 4}$. We can join these two components by a Jordan $\operatorname{arc} L_{n}$ in $\tilde{D}_{n}$ where $\pi\left(L_{n}\right)$ is a segment $\left[a_{n}, s_{n}\right]$. Then $D_{n}=\tilde{D}_{n} \backslash L_{n}$ satisfies all conditions of (d) if we remove "a neighborhood of" in the condition (iv) of (d).

\section{References}

[1] Bishop E., Analyticity in certain Banach algebras. Trans. Amer. Math. Soc. 102 (1962), 507-544.

[2] Gamelin T.W. and Hayashi M., The algebra of bounded analytic functions on a Riemann surface. J. Reine Angew. Math. 382 (1987), 49-73.

[3] Myrberg P.J., Über die analytische Fortsetzung von beschränkten Funktionen. Ann. Acad. Sci. Fenn. Ser A.I. No. 58 (1949), p. 7.

[4] Royden H.L., Algebras of bounded analytic functions on Riemann surfaces. Acta Math. 114 (1965), 113-142.

Department of Mathematics

Daido Institute of Technology

Takiharu, Minami, Nagoya 457-8530

Japan

narita@daido-it.ac.jp 